This is the accepted version of

Horváth, J. (2014) Sensory ERP effects in auditory distraction: did we miss the main event? Psychological Research 78, 339-348. doi: 10.1007/s00426-013-0507-7

The final publication is available at link.springer.com:

http://link.springer.com/article/10.1007\%2Fs00426-013-0507-7

\title{
Sensory ERP effects in auditory distraction: did we miss the
}

\section{main event?}

\author{
János Horváth
}

Institute of Cognitive Neuroscience and Psychology, RCNS, Hungarian Academy of Sciences, Budapest, Hungary

\section{Address correspondence to:}

János Horváth

Institute of Cognitive Neuroscience and Psychology, RCNS, HAS

P.O.B. 398, Szondi u $83 / 85$

H-1394 Budapest

HUNGARY

Phone: +3613542290

Fax: +3613542416

E-mail: horvath.janos@ttk.mta.hu

28 pages (including 4 figures) 


\section{AbSTRACT}

Event-related potentials (ERPs) offer unique insights into processes related to involuntary attention changes triggered by rare, unpredictably occurring sensory events, that is, distraction. Contrasting ERPs elicited by distracters and frequent standard stimuli in oddball paradigms allowed the formulation of a three-stage model describing distractionrelated processing: First, the distracting event is highlighted by a sensory filter. Second, attention is oriented towards the event, and finally, the the task-optimal attention set is restored, or task priorities are changed. Although this model summarizes how distracting stimulus information is processed, not much is known about the cost of taking this exceptional route of processing. The present study demonstrates the impact of distraction on sensory processing. Participants performed a Go/NoGo tone-duration discrimination task, with infrequent pitch distracters. In the two parts of the experiment the duration-response mapping was reversed. Contrasts of distracter and standard ERPs revealed higher P3a- and reorienting negativity amplitudes for short than for long tones, independently from response type. To understand the cause of these asymmetries, short vs. long ERP contrasts were calculated. The ERP pattern showed that short standards elicited an attention-dependent offset- response, which was abolished for short distracters. That is, the apparent P3a- and RON enhancements were caused by the removal of a task-related attentional sensory enhancement. This shows that the disruption of task-optimal attention set precedes the elicitation of the P3a, which suggests that $\mathrm{P} 3 \mathrm{a}$ does not reflect a process driving the initial distraction-related attention change. 


\section{INTRODUCTION}

Distraction, the involuntary attention change triggered by unpredictably occurring sensory events has been the focus of numerous studies in the last two decades. Most of these studies relied on variations of the oddball paradigm and the registration of event-related brain potentials (ERPs). Their results delineated a three-stage model describing the unfolding of distraction-related processing (Escera \& Corral, 2003; Escera, Alho, Schröger, Winkler, 2000; Horváth, Winkler, Bendixen, 2008). The first stage is a sensory filter, which highlights informative (distracting) sensory events. The second stage features modality-independent processes which allocate attention to such events. Finally, on the third stage, the processing of the distracting event is concluded by the restoration of the task-optimal attention set, or the change of task priorities (e.g., we continue doing what we have been doing before, or change our goals and behavior to match the changed circumstances). Whereas this model elegantly summarizes how the cognitive system processes distracting stimulus information, the cost of taking this exceptional route of processing is not described. That is, the consequences of distraction on the cognitive system remain largely unspecified. The present study demonstrates the sensory impact of distraction: it is shown that after the sensory filter highlights the distracter, sensory processing does not immediately resume operation as before. That is, although the distraction-related "processing focus" moves on to higher stages, the effects of distraction still persist on the level of sensory processing.

This insight on the sensory effects of distraction is made possible by investigating a somewhat particular question regarding a variation of the oddball paradigm introduced by Schröger \& Wolff (the distraction paradigm, 1998b). In this paradigm, participants perform a two-alternative tone-duration discrimination task: $50 \%$ of the presented tones are short (e.g., $200 \mathrm{~ms}$ ), 50\% are long (e.g., $400 \mathrm{~ms}$ ). Whereas most of the tones have the same pitch (these are termed standards), unpredictably, typically $10-20 \%$ of the time, the pitch of a tone is 
changed (independently from its duration; these tones are termed deviants). Because

participants perform the same task for deviants as for standards, it is assumed that differences in (behavioral or ERP) responses to deviants and standards reflect processes related solely to distraction.

In studies using the distraction paradigm, the comparison of ERPs to deviants and standards showed a consistent pattern of responses assumed to reflect the three stages of distraction-related processing summarized above (e.g., Schröger \& Wolff, 1998a; Berti \& Schröger, 2001, Roeber, Berti, Schröger, 2003). Following the deviance-onset, processing at the sensory filter is reflected by frontal and central negative differences between 100-200 ms, a superposition of an N1-effect (Näätänen \& Picton, 1987), mismatch negativity (Näätänen, Gaillard \& Mäntysalo, 1978, for a recent summary see Winkler, 2007), and possibly N2(b) (Näätänen, Simson, \& Loveless, 1982; Ritter et al., 1992). The allocation of attention at the second stage is reflected by the P3a peaking fronto-centrally around $300 \mathrm{~ms}$ (Friedman, Cycowicz, \& Gaeta, 2001), and re-orientation at the third stage is reflected by the so-called reorienting negativity (RON, Schröger \& Wolff, 1998a) observable fronto-centrally in the 400-600 ms interval.

Although in this paradigm participants consistently make more errors in deviant trials, and response times to deviants are delayed in comparison to standards (e.g., Roeber, Widmann, Schröger, 2003) these differences are not "pure" measures of distraction. The reaction time difference sums a number of effects, some of which are unrelated to distraction: differences in the alerting and fore-period effects (Parmentier, Elsley, Ljungberg, 2010; Ljungberg, Parmentier, Leiva, \& Vega, 2012; Wetzel, Widmann, Schröger, 2012; Li, Parmentier, Zhang, 2013, Wetzel, Schröger, \& Widmann, in press) of the deviant and standard stimulus events may even result in deviance-related speedups in certain setups . For example, if stimuli are presented at a slow rate, a feature-rich, novel deviant preceding the 
task-relevant stimulus event might substantially raise the participant's level of arousal in comparison to standard trials, which may enhance response speed (SanMiguel, Linden, \& Escera, 2010; SanMiguel, Morgan, Klein, Linden, \& Escera, 2010).

Because most studies focused on distraction-related processing, they did not separate short and long tone trials in the analyses. Short and long trials were separately analyzed, however, in the study by Horváth, Czigler, Birkás, Winkler and Gervai (2009). They used a distraction paradigm with a 150 vs. $600 \mathrm{~ms} \mathrm{Go/NoGo} \mathrm{duration-discrimination} \mathrm{task} \mathrm{and} \mathrm{rare}$ pitch-deviances. It was found that in short (Go) trials higher amplitude P3a and RON were elicited than in long (NoGo) trials (as observable in the deviant-minus-standard difference waveforms). Because the distracting feature was the same for both short and long stimuli, these differences cannot be explained in the three-stage model: For example, why would a short deviant be more distracting (as signaled by higher amplitude P3a and RON) than a long deviant? This result may indicate that the three-stage model has to be extended, or the functional interpretation of $\mathrm{P} 3 \mathrm{a}$ and $\mathrm{RON}$ has to be revised.

One possibility for extending the model is to assume that distracters affect not only processes related to the orientation of attention, but also processes related to response organization. This is hinted at by a couple of previous studies: Roeber, Berti, Widmann and Schröger (2005) found that P3a and RON were sensitive to local response sequences, that is, tones requiring a response repetition elicited lower amplitude P3a and RON than those requiring a response change (see also Roeber, Berti, Müller, Widmann \& Schröger, 2009). The studies by Escera, Yago, \& Alho (2001), Munka \& Berti (2006), and Horváth, Maess, Berti \& Schröger (2008) also open up the possibility that RON may in part reflect activities related to motor- or task-preparation. ( Based on these results, one may speculate that the short (Go) vs. long (NoGo) asymmetry in the Horváth et al. (2009) study might be due to the task-role (Go/NoGo) of the stimuli. 
As an alternative, Horváth et al. (2009) suggested that P3a and RON modulations may also reflect an additional tone-offset-related ERP. Because attention is known to enhance tone-onset-related auditory ERPs (e.g., Hillyard, Hink, Schwent \& Picton, 1973; Okamoto, Stracke, Wolters, Schmael \& Pantev, 2007), it seems quite possible that attention may also enhance offset-related ERP responses as well.

However, because duration and task-role was perfectly confounded in the Horváth et al. (2009) experiment, the differences could not be unequivocally attributed to the duration or the task-role of the tones. In the present study, through the independent variation of duration (short/long) and task role (Go/NoGo) in a distraction paradigm closely matching that of Horváth et al. (2009), the source of asymmetries in the distraction-related ERPs were investigated. In the initial analyses ERPs were identified on the basis of deviant vs. standard contrasts. To better understand the asymmetries found in these contrasts, further ERP analyses based on short-minus-long differences were also conducted, which revealed an effect interpretable as an attention-dependent sensory effect.

\section{METHODS}

\section{Participants}

Sixteen healthy volunteers (twelve women; age 20-28 years, mean: 24 years; all righthanded) participated in the experiment for course credit or monetary compensation. All participants reported normal hearing status and gave written informed consent after the experimental procedures were explained to them. None of them participated in the Horváth et al. (2009) study.

\section{Stimuli and procedure}

Participants were seated in a comfortable chair in an acoustically and electrically shielded room during the experiment. Sequences of tones were presented through headphones 
(Sennheiser HD-25, Wedemark, Germany). As in the study by Horváth et al. (2009) the tones were composed of three sinusoids: Standards had a fundamental frequency of $700 \mathrm{~Hz}$, low-, and high deviants 636 and $770 \mathrm{~Hz}$, respectively. The two overtones were one and two octaves above the fundamental frequency, with relative intensities of -3 and $-6 \mathrm{~dB}$, respectively. Tone intensity was $70 \mathrm{~dB}$ (A-weighted). Stimulus duration was $150 \mathrm{~ms}$ for short, and $600 \mathrm{~ms}$ for long tones (including $2.5-2.5 \mathrm{~ms}$ rise and fall times). The onset-to-onset inter-stimulus interval was $1400 \mathrm{~ms}$.

Participants performed a Go/NoGo task in two conditions: in the first condition, they were instructed to press a button held in their dominant hand for short stimuli, but hold response for the long ones (irrespective of their pitch). In the second condition, the keypressduration association was reversed. The conditions were presented in the first and second halves of the experimental session. The order of the conditions was counterbalanced between participants. The instruction emphasized both response speed and accuracy. Before mounting the electrodes, participants performed the task in a practice block of thirty trials in which all combinations of sound durations and pitches were presented equiprobably.

Each condition was divided into eleven experimental blocks, each comprising 112 standards (58-58 long and short) and 16 deviants (4 short-high, 4 long-high, 4 short-low, and 4 long-low), presented in a random order. That is, deviant frequency was $12.5 \%$.

\section{EEG recording and analysis}

EEG was recorded using $\mathrm{Ag} / \mathrm{AgCl}$ electrodes with an Active-Two system (BioSemi, Amsterdam, The Netherlands), with $512 \mathrm{~Hz}$ sampling rate from the scalp-locations Fp1, Fp2, AF3, AF4, F7, F3, Fz, F4, F8, FC5, FC1, FC2, FC6, T7, C3, Cz, C4, T8, CP5, CP1, CP2, CP6, P7, P3, Pz, P4, P8, PO3, PO4, O1, Oz, O2 (according to the 10\% system, Nuwer et al, 1998). Additional electrodes were placed on the mastoids (left: Lm and right: Rm). The 
reference electrode (for off-line re-referencing) was placed on the tip of the nose. Horizontal eye-movements were monitored with electrodes placed lateral to the outer canthi of the two eyes; vertical eye-movements were monitored with electrodes placed above and below the right eye.

The EEG was offline $0.25-20 \mathrm{~Hz}$ bandpass filtered (Kaiser-windowed sinc finite impulse response filter, beta of 4.55, 3749 coefficients; transition bandwidth $0.4 \mathrm{~Hz}$, stopband attenuation at least $50 \mathrm{~dB}$ ). For each trial, epochs of $1100 \mathrm{~ms}$ were extracted including a 200 ms pre-stimulus interval. The average signal measured in the pre-stimulus interval served as a baseline for the amplitude calculations. Epochs corresponding to the first four trials of each block, and those with a signal range exceeding $150 \mu \mathrm{V}$ on any channel were discarded from the analyses. Epochs were separately averaged for each combination of frequency (deviant or standard), duration (short or long), and task role (Go or NoGo).

For the ERP analyses two approaches were taken. For the first approach, as usual in the literature, deviant-minus-standard difference waveforms were calculated. The peak latencies of the distraction-related ERPs were identified in the group-average short Go deviant-minus-standard difference waveform (for which the highest P3a and RON amplitudes were expected, based on Horváth et al., 2009), at frontal and central sites. The average signals in $20 \mathrm{~ms}$ long windows centered at these latencies were used to calculate individual amplitudes for the N1-effect, P3a and RON at Fz. For the N2, amplitudes were measured at Cz. The amplitudes were submitted to repeated-measures analyses of variance (ANOVAs) with Frequency (standard or deviant), Task Role (Go or NoGo) and Duration (long or short) factors. In these analyses, the main question was whether an interaction including Frequency was present, because this would indicate that the amplitude of the distraction-related ERP was modulated by Task Role or Duration. The alpha-level was set to .05. Generalized eta-squared 
effect sizes (Olejnik and Algina, 2003; Bakeman, 2005) are reported. Some interactions were resolved using pair-wise, two-tailed, paired Student's t-tests.

Because the first approach showed that the amplitudes of the ERPs observable in the deviant-minus-standard difference waveform were modulated by Duration, in the second approach short-minus-long difference waveforms were calculated separately for standards and deviants in order to find a possible explanation for these interaction patterns. The shortminus-long differences for standards exhibited a triphasic (positive-negative-positive) waveform which was identified as the succession of an offset-related P1, N1, and P2. Individual amplitudes were calculated as average signals in $20 \mathrm{~ms}$ long windows centered at the latencies of these group-average waveforms, and submitted to Frequency $\times$ Duration repeated measures ANOVAs.

d' sensitivity scores and reaction times were analyzed in Frequency (standard or deviant) $\times$ Condition (Short-Go or Long-Go) repeated measures ANOVAs. For these analyses only 200-1300 ms intervals following tone-onsets were taken into account. To avoid infinities in the d' calculations, hit rates of 1 and false alarm rates of zero were adjusted to $1-(1 /(2 \mathrm{~N}))$ and $1 /(2 \mathrm{~N})$, respectively (where $\mathrm{N}$ is the number of hits and correct rejections, respectively). Because in these calculations a higher number of trials may lead to biased d' scores, the numbers of hits, misses, correct rejections and false alarms for standards were proportionally scaled down to match the number of deviant trials and rounded to the nearest integer (see Macmillan and Creelman, 2005). To better specify the cause of d' effects, hit rates and false alarm rates were analyzed similarly.

For reaction times (calculated only for correct responses), it is important to note that in this analysis Condition is perfectly confounded with tone Duration. The Go-NoGo duration discrimination task has an inherent asymmetry: In the Short-Go condition the decision to respond is triggered by the detection of the transient, whereas in the Long-Go condition this 
decision is based on the assessment whether sufficient time has passed to be sure that the given tone is long (i.e. that the transient would have occurred if the tone would have been short). This may lead to between-condition differences in response criteria, which may contribute to the ANOVA effects including the Condition factor. Individuals were characterized by the median of the reaction times measured in the respective trials. The first four trials of each block were omitted from these analyses.

\section{RESULTS}

\section{Behavioral data}

The Frequency $\times$ Condition ANOVA of the d' scores times showed a Frequency main effect: $\mathrm{F}(1,15)=8.06, \eta_{\mathrm{G}}{ }^{2}=0.06, \mathrm{p}=.01$; and a Condition main effect: $\mathrm{F}(1,15)=10.12, \eta_{\mathrm{G}}{ }^{2}=$ $0.07, \mathrm{p}=.006$, showing that $\mathrm{d}$ 's were lower for deviants than for standards, and lower in the Long-Go than in the Short-Go condition (Figure 1, left). This between-condition difference is on-a-par with the assumption of an inherent between-condition difference described above: if overt responses are contingent on the assessment whether enough time has passed to be sure that the current tone is long, then the motivation to respond as fast as possible will lead to more false alarms (too early decisions to respond) in this situation. This interpretation is supported by the Frequency $\times$ Condition ANOVA of the false alarm rates, which showed a Frequency main effect: $\mathrm{F}(1,15)=8.58, \eta_{\mathrm{G}}{ }^{2}=0.05, \mathrm{p}=.01$; and a Condition main effect: $\mathrm{F}(1,15)=6.12, \eta_{\mathrm{G}}{ }^{2}=0.06, \mathrm{p}=.03$, showing that false alarm rate was higher in deviant than in standard trials (4.3 vs. $2.8 \%$ ), and higher in the Long-Go than in the Short-Go ( $4.4 \%$ vs. $2.7 \%$ ) condition. The same type of analysis for hit rates showed no significant effects. The Frequency $\times$ Condition ANOVA of the reaction times showed a Frequency main effect: $\mathrm{F}(1,15)=73.93, \eta_{\mathrm{G}}{ }^{2}=0.05, \mathrm{p}<.001 ;$ a Condition main effect: $\mathrm{F}(1,15)=17.34, \eta_{\mathrm{G}}{ }^{2}=0.10$, $\mathrm{p}<.001$; and a Frequency $\times$ Condition interaction: $\mathrm{F}(1,15)=37.47, \eta_{\mathrm{G}}{ }^{2}=0.01, \mathrm{p}<.001$, 
showing that the reaction time delay for deviants was longer in the Long-Go than in the ShortGo condition (Figure 1, right).

\section{Event-related potentials}

The group-average ERPs and the corresponding deviant-minus standard difference waveforms elicited at midline sites and the average mastoid signals are presented in Figure 2. The short Go deviant-minus-standard difference waveform exhibited an N1-effect peaking at F4 at $109 \mathrm{~ms}, \mathrm{~N} 2$ peaking at $\mathrm{Cz}$ at $181 \mathrm{~ms}, \mathrm{P} 3 \mathrm{a}$ peaking at $\mathrm{Fz}$ at $318 \mathrm{~ms}$, and RON peaking at FC1 at 416 ms. (Note that the N1-effect and RON peaks were not at the predetermined electrode of interest - Fz, but still at neighboring electrodes. For the N1-effect, the peak latency was the same at F4 and Fz; for RON, it was 2 ms later at Fz). The ANOVA of the amplitudes in the N1-interval showed a significant Frequency main effect: $F(1,15)=37.62$, $\eta_{\mathrm{G}}{ }^{2}=0.12, \mathrm{p}<.001$, showing that deviants elicited higher (more negative) N1s than standards.

The ANOVA of the amplitudes in the N2-interval at $\mathrm{Cz}$ showed a significant Frequency main effect: $\mathrm{F}(1,15)=4.98, \eta_{\mathrm{G}}{ }^{2}=0.03, \mathrm{p}=.04$, showing higher (more negative) amplitudes for deviants than standards.

The ANOVA of the amplitudes in the P3a-interval at Fz showed a Frequency main effect: $\mathrm{F}(1,15)=57.29, \eta_{\mathrm{G}}{ }^{2}=0.34, \mathrm{p}<.001 ;$ a Duration main effect: $\mathrm{F}(1,15)=14.83, \eta_{\mathrm{G}}{ }^{2}=$ $0.05, \mathrm{p}=.002$; a Task Role main effect: $\mathrm{F}(1,15)=7.14, \eta_{\mathrm{G}}{ }^{2}=0.01, \mathrm{p}=.02$; and a Frequency $\times$ Duration interaction: $\mathrm{F}(1,15)=44.19, \eta_{\mathrm{G}}{ }^{2}=0.04, \mathrm{p}<.001$. The Task Role main effect showed that the tones requiring an overt response elicited higher (more positive) amplitudes than those which did not. The Frequency $\times$ Duration interaction (see Figure 3, left) shows that the deviant-minus-standard difference was larger (more positive) for short tones. For the Frequency $\times$ Duration interaction, pair-wise $t$-tests between the corresponding four 
amplitudes (six comparisons) showed significant differences in all comparisons (t[15]-values $>5.52$ and $\mathrm{p}$-values $<.001)$, but the one between deviants $(\mathrm{t}[15]=0.54, \mathrm{p}=0.60)$.

The ANOVA of the amplitudes in the RON-interval at Fz showed a Frequency main effect: $\mathrm{F}(1,15)=4.98, \eta_{\mathrm{G}}{ }^{2}=0.02, \mathrm{p}=.04$; a Duration main effect: $\mathrm{F}(1,15)=22.07, \eta_{\mathrm{G}}{ }^{2}=0.09$, $\mathrm{p}<.001$; and a Frequency $\times$ Duration interaction: $\mathrm{F}(1,15)=14.10, \eta_{\mathrm{G}}{ }^{2}=0.04, \mathrm{p}=.002$, showing that for short tones, a larger (more negative) RON (deviant-minus-standard difference) was elicited than for long tones. Pair-wise t-tests between the corresponding four amplitudes (six comparisons, see Figure 3, right) showed that the short standard significantly differed from all others $(\mathrm{t}[15]$-values $>3.58$ and $\mathrm{p}$-values $<.003)$, and there was a significant difference between the short deviant and the long standard as well $(\mathrm{t}[15]=2.27, \mathrm{p}=0.04)$.

To understand the cause of interactions in the P3a and RON time intervals (see Figure 3, left and middle column, and also the Discussion), ERPs to short and long tones were contrasted separately for deviants and standards (Figure 3, right column). The standard shortminus-long difference waveform exhibited a positivity peaking at $\mathrm{Cz}$ at $248 \mathrm{~ms}$ (after tone onset), a negativity peaking at $\mathrm{Fz}$ at $310 \mathrm{~ms}$, and a positivity peaking at $\mathrm{Cz}$ at $453 \mathrm{~ms}$, followed by a slow trend towards the baseline. These were identified as offset-related P1, N1, and the superposition of $\mathrm{P} 2$ and duration-dependent sustained activity (see Discussion). For the amplitudes (average signals in $20 \mathrm{~ms}$ long intervals centered at the peak latencies) Frequency $\times$ Duration ANOVAs were calculated at the peak electrode.

The ANOVA of the amplitudes in the offset-P1 interval at $\mathrm{Cz}$ showed a Frequency main effect: $\mathrm{F}(1,15)=13.26, \eta_{\mathrm{G}}{ }^{2}=0.05, \mathrm{p}=.002$; and a Duration main effect: $\mathrm{F}(1,15)=5.66$, $\eta_{\mathrm{G}}{ }^{2}=0.02, \mathrm{p}=.03$, indicating that ERPs to standards, and short tones were higher (more positive) in this interval. 
Unsurprisingly (because the offset-N1 interval overlaps the P3a interval), the ANOVA of the amplitudes in the offset-N1 interval at Fz showed a Frequency main effect: $F(1,15)=$ 46.09, $\eta_{\mathrm{G}}{ }^{2}=0.36, \mathrm{p}<.001$; a Duration main effect: $\mathrm{F}(1,15)=17.97, \eta_{\mathrm{G}}{ }^{2}=0.07, \mathrm{p}<.001$; and a Frequency $\times$ Duration interaction: $\mathrm{F}(1,15)=36.34, \eta_{\mathrm{G}}{ }^{2}=0.04, \mathrm{p}<.001$, indicating that the short-minus-long difference was higher (more negative) for standards than for deviants.

The ANOVA of the amplitudes in the offset-related P2-interval at $\mathrm{Cz}$ showed a Duration main effect: $\mathrm{F}(1,15)=49.14, \eta_{\mathrm{G}}{ }^{2}=0.10, \mathrm{p}<.001$; and a Frequency $\times$ Duration interaction: $\mathrm{F}(1,15)=8.31, \eta_{\mathrm{G}}{ }^{2}=0.01, \mathrm{p}=.01$, indicating that the short-minus-long difference was higher (more positive) for standards than for deviants.

\section{DisCUSSION}

In general, the results are consistent with previous studies using the distractionparadigm: Reaction times were longer, and discrimination performance decreased for deviants, and the results are consistent with the inherent asymmetry of the Go-NoGo duration discrimination task: lower sensitivity scores were caused by higher false alarms rates (too early decisions to respond) in the Long-Go condition. Whereas reaction time delays differed between tone durations, the fact that reaction times for the short and long tones came from different conditions does not allow an unequivocal interpretation of this difference. Deviantminus-standard waveforms exhibited negativities between 100-200 ms following deviance onset (a superposition of an N1-effect, MMN and N2), P3a around $320 \mathrm{~ms}$, and RON around $420 \mathrm{~ms}$. The present study replicated the findings by Horváth et al. (2009): deviant-minusstandard ERP amplitudes in the P3a and RON intervals were higher for short than for long tones. Furthermore, the task role of the tones (i.e. whether one had to make an overt response based on duration or not) did not substantially modulate these ERPs. 
Although in general the results are in agreement with previous studies, explaining the duration-dependency of the ERP amplitudes in the P3a and RON intervals is not trivial. Because the distracting event is the same for short and long stimuli, P3a and RON amplitudes should not differ between tone durations. This discrepancy can be resolved by assuming that the amplitude-effects are not genuine modulations of P3a and RON, rather, that they are caused by an additional ERP component which is present in only one of the conditions. Horváth et al. (2009) suggested that this additional ERP could be a 1) tone-offset-related ERP, which is 2) observable only when participants attend the offset.

The tone offset-related ERP could be made visible by contrasting the standard ERPs: The short-minus-long standard difference waveform shows an auditory transient-related ERP response (Figure 3, right column): it starts with a positivity peaking centrally at around 100 $\mathrm{ms}$ following the offset (250 ms after tone onset), followed by a negativity at about $150 \mathrm{~ms}$ (300 ms after tone onset), and a positive peak at around $300 \mathrm{~ms}$ (450 ms after tone onset). Despite delayed by about $50 \mathrm{~ms}$ compared to an onset-related auditory ERP response, these waveforms can be identified as the succession of P1, N1, and P2. Note that the offset-related $\mathrm{P} 2$ is followed and probably overlapped by a slow, sustained positive waveform.

In comparison, in the short-minus-long deviant difference waveform the offset-related ERP response (Figure 3, right) is largely missing. This can be interpreted as a distractionrelated removal of attention, which reduces (and in this case even abolishes) the offset-related ERP response similarly to that reported by Horváth and Winkler (2010) in the context of a gap-detection task (in which a rare frequency glide preceding the task-relevant gap by about $150 \mathrm{~ms}$ resulted in strongly reduced gap-related N1 and P2). The short-minus-long deviant difference waveform showed a hint at a P1, and a sustained fronto-central positivity starting around $400 \mathrm{~ms}$ following tone onset and ending around $700 \mathrm{~ms}$. The analyses of the offset-P1 amplitudes showed no significant distraction-related modulation, so it is possible that $\mathrm{P} 1$ is 
not affected by deviance, but it is also likely that the power of the present study was insufficient to resolve this potential effect. The sustained activity can be identified as the reversed, duration-dependent sustained negativity which is present for long, but not for short tones. This is quite similar to that observable in the difference of the standards overlapping and following the offset-related P2 peak.

For the ERPs in the P3a interval, the attention-dependent offset-response-based explanation fits the results perfectly. The amplitude analyses showed that the enhancement stemmed not from a differential ERP response to short and long deviants, rather, it stemmed from a difference between ERPs to short and long standards: The attention-dependent offsetN1 was present for short standards at the latency of the P3a, but this N1 was strongly reduced for short deviants, because the preceding deviance removed the attentional enhancement of the offset response. Because in the deviant-minus-standard waveforms the offset-related (negative) N1 is subtracted, the subtraction increases the amplitude of the positive difference waveform in the P3a interval.

For the ERPs in the RON interval, a similar line of thought can be formulated with the attention-dependent offset-related P2. Here the subtraction of the positivity in the deviantminus-standard waveform results in a negative ERP enhancement in the RON interval. Note that whereas the amplitude analyses in the P3a interval suggest that a component was superimposed on the P3a, in the RON interval, an underlying component (RON) was not observable. That is, the present results cannot rule out that RON may simply be an attentiondependent modulation of the offset-related P2 (with reversed polarity due to the subtraction) in the present design.

The two ways of comparing the ERPs (short-minus-long vs. deviant-minus-standard) offer partially different views on distraction-related ERP elicitation patterns. Whereas one 
could argue that the short vs. long comparison revealed only effects already observable in the deviant-minus-standard waveform, a number of arguments support the notion that the contrast enables a more economic explanation to these effects: First, the duration-dependent differences are incompatible with the assumptions of the original framework, which is based on the deviant-minus-standard comparison. Second, the short-minus-long difference revealed a well-recognizable ERP pattern characteristic of transient-related auditory ERPs. If, for example, the short-minus-long standard waveform would have shown only a single negative peak at the P3a latency, which would not be present for the difference of the deviants, one could argue, that this might simply reflect a coincidence resulting from the specific parameters of the experiment. The chance, however, that such a consistent triphasic waveform would be simply a coincidence, is probably quite slim. Third, the present study is not the first to suggest that the task-relevant sensory event following deviance onset may trigger loweramplitude sensory ERPs than those following the standard events. Beside the study by Horváth \& Winkler (2010), Schröger (1996) already suggested this possibility.

These results have profound impact on the functional interpretation of P3a. Although there is no general consensus on the functional interpretation of the P3a (Dien, Spencer, \& Donchin, 2004), results of oddball paradigms suggested that P3a was related to attentionchanges triggered by unpredictably occurring, rare stimulus variations (Friedman, Cycowicz, Gaeta, 2001). The results of the present study strongly constrain this notion because the offset-related N1 peak that should have been elicited by the short deviant was abolished by the time the P3a was elicited. This means that the task-relevant attention set (which would be reflected by an offset-related ERP) was already disrupted before the P3a was elicited, which implies that the process reflected by P3a does not drive the initial attention change, that is, P3a does not reflect involuntary attention change (see also Barcelo, Escera, Corral \& 
Periáñez, 2006; Horváth, Winkler, Bendixen, 2008; for a recent summary, see Wetzel et al., in press).

The nature of the initial attention change is unclear in the present paradigm: the results show only that the offset-focused attention is gone by about $150 \mathrm{~ms}$ after deviance onset. It is possible that a different (possibly pitch-instead of duration-focused) attention set has been already established, but it is also possible that a general resetting of attention might have happened (see Horváth, Roeber, Bendixen, \& Schröger, 2008). Based on the present results, P3a may still reflect a process related to the mobilization of attentional resources as part of the orienting response towards the distracting stimulus feature, that is, it may be related to establishing an attention set focused on pitch in the present case. Because the present study also casts some doubts on the assumption that RON (as observed in the present paradigm) is an independent component, one may also speculate that P3a might actually reflect reorienting, i.e. re-establishing the task-optimal (offset-focused) attention set.

In summary, the separation of short and long tones in the analyses led to an insight on the sensory impact of distraction. Contrasting ERPs to short and long tones showed that distraction abolished attention-dependent sensory ERPs reflecting the augmented processing of the task-relevant auditory transient. This effect persisted for at least $150 \mathrm{~ms}$ following the distracting event. 


\section{ACKNOWLEDGEMENTS}

The experiment was conducted at the University of Leipzig, Institute for Psychology I, in the Cognitive including Biological Psychology Research Group. The study was supported the European Commission under the Marie Curie Intra-European Fellowship Project MEIFCT-2006-023924, and the János Bolyai Research Scholarship of the Hungarian Academy of Sciences. The experiment was realized using Cogent 2000 developed by the Cogent 2000 team at the FIL and the ICN. I thank Jenny Kokinous for assistance in data collection. I also thank three anonymous reviewers for constructive comments and critiques. 


\section{REFERENCES}

Bakeman, R. (2005). Recommended effect size statistics for repeated measures designs. Behavior Research Methods, 37(3), 379-384. doi:10.3758/BF03192707

Barcelo, F., Escera, C., Corral, M. J., \& Periáñez, J. A. (2006). Task switching and novelty processing activate a common neural network for cognitive control. Journal of Cognitive Neuroscience, 18(10), 1734-1748.

Berti, S., Roeber, U., \& Schröger, E. (2004). Bottom-up influences on working memory: behavioral and electrophysiological distraction varies with distractor strength. Experimental psychology, 51(4), 249-257.

Berti, S., \& Schröger, E. (2001). A comparison of auditory and visual distraction effects: behavioral and event-related indices. Cognitive brain research, 10(3), 265-273.

Dien, J., Spencer, K. M., \& Donchin, E. (2004). Parsing the late positive complex: Mental chronometry and the ERP components that inhabit the neighborhood of the P300. Psychophysiology, 41(5), 665-678. doi:10.1111/j.1469-8986.2004.00193.x

Escera, C., Alho, K., Schröger, E., \& Winkler, I. (2000). Involuntary attention and distractibility as evaluated with event-related brain potentials. Audiology and Neurotology, 5(3-4), 151-166.

Escera, C., \& Corral, M.-J. (2003). The distraction potential (DP), an electrophysiological tracer of involuntary attention control and its dysfunction. The cognitive neuroscience of individual differences, 63-76.

Escera, C., Yago, E., \& Alho, K. (2001). Electrical responses reveal the temporal dynamics of brain events during involuntary attention switching. European Journal of Neuroscience, 14(5), 877-883.

Friedman, D., Cycowicz, Y. M., \& Gaeta, H. (2001). The novelty P3: an event-related brain potential (ERP) sign of the brain's evaluation of novelty. Neuroscience and biobehavioral reviews, 25(4), 355-373. 
Hillyard, S. A., Hink, R. F., Schwent, V. L., \& Picton, T. W. (1973). Electrical signs of selective attention in the human brain. Science, 182(4108), 177-180. doi:10.1126/science.182.4108.177Horváth, J., Czigler, I., Birkás, E., Winkler, I., \& Gervai, J. (2009). Age-related differences in distraction and reorientation in an auditory task. Neurobiology of Aging, 30(7), 1157-1172. doi:10.1016/j.neurobiolaging.2007.10.003

Horváth, J., Maess, B., Berti, S., \& Schröger, E. (2008). Primary motor area contribution to attentional reorienting after distraction. Neuroreport, 19(4), 443-446.

Horváth, J., Winkler, I., \& Bendixen, A. (2008). Do N1/MMN, P3a, and RON form a strongly coupled chain reflecting the three stages of auditory distraction? Biological Psychology, 79(2), 139-147. doi:10.1016/j.biopsycho.2008.04.001

Kushnerenko, E., Fellman, V., Huotilainen, M., \& Winkler, I. (2001). Event-related potential correlates of sound duration: similar pattern from birth to adulthood. NeuroReport, 12(17), 3777-3781.

Li, B., Parmentier, F. B. R., \& Zhang, M. (2013). Behavioral distraction by auditory deviance is mediated by the sound's informational value. Experimental Psychology, 60, 260268. doi:10.1027/1618-3169/a000196

Ljungberg, J. K., Parmentier, F. B. R., Leiva, A., \& Vega, N. (2012). The informational constraints of behavioral distraction by unexpected sounds: The role of event information. Journal of Experimental Psychology: Learning, Memory, and Cognition, 38, 1461-1468. doi:10.1037/a0028149Macmillan, N. A., \& Creelman, C. D. (2005). Detection theory: a user's guide (2nd ed.). Mahwah, N.J: Lawrence Erlbaum Associates.

Munka, L., \& Berti, S. (2006). Examining task-dependencies of different attentional processes as reflected in the P3a and reorienting negativity components of the human event- 
related brain potential. Neuroscience Letters, 396(3), 177-181.

doi:10.1016/j.neulet.2005.11.035

Näätänen, R., Gaillard, A. W., \& Mäntysalo, S. (1978). Early selective-attention effect on evoked potential reinterpreted. Acta psychologica, 42(4), 313-329.

Näätänen, Risto, \& Picton, T. (1987). The N1 wave of the human electric and magnetic response to sound: A review and an analysis of the component structure. Psychophysiology, 24(4), 375-425. doi:10.1111/j.1469-8986.1987.tb00311.x

Näätänen, Risto, Simpson, M., \& Loveless, N. E. (1982). Stimulus deviance and evoked potentials. Biological psychology, 14(1), 53-98.

Okamoto, H., Stracke, H., Wolters, C. H., Schmael, F., \& Pantev, C. (2007). Attention improves population-level frequency tuning in human auditory cortex. Journal of Neuroscience, 27(39), 10383-10390. doi:10.1523/JNEUROSCI.2963-07.2007

Olejnik, S., \& Algina, J. (2003). Generalized eta and omega squared statistics: Measures of effect size for some common research designs. Psychological Methods, 8(4), 434-447. doi:10.1037/1082-989X.8.4.434

Parmentier, F. B. R., Elsley, J. V., \& Ljungberg, J. K. (2010). Behavioral distraction by auditory novelty is not only about novelty: The role of the distracter's informational value. Cognition, 115(3), 504-511. doi:10.1016/j.cognition.2010.03.002

SanMiguel, I., Linden, D., \& Escera, C. (2010). Attention capture by novel sounds:

Distraction versus facilitation. European Journal of Cognitive Psychology, 22(4), 481515. doi:10.1080/09541440902930994

Ritter, W., Paavilainen, P., Lavikainen, J., Reinikainen, K., Alho, K., Sams, M., \& Näätänen, R. (1992). Event-related potentials to repetition and change of auditory stimuli. Electroencephalography and clinical Neurophysiology, 83(5), 306-321. 
Roeber, U., Berti, S., Müller, D., Widmann, A., \& Schröger, E. (2009). Disentangling effects of auditory distraction and of stimulus-response sequence. Psychophysiology, 46(2), 425-438. doi:10.1111/j.1469-8986.2008.00766.x

Roeber, U., Berti, S., \& Schröger, E. (2003). Auditory distraction with different presentation rates: An event-related potential and behavioral study. Clinical Neurophysiology, 114(2), 341-349.

Roeber, U., Berti, S., Widmann, A., \& Schröger, E. (2005). Response repetition vs. response change modulates behavioral and electrophysiological effects of distraction. Cognitive Brain Research, 22(3), 451-456. doi:10.1016/j.cogbrainres.2004.10.001

Roeber, U., Widmann, A., \& Schröger, E. (2003). Auditory distraction by duration and location deviants: a behavioral and event-related potential study. Cognitive Brain Research, 17(2), 347-357.

SanMiguel, I., Morgan, H. M., Klein, C., Linden, D., \& Escera, C. (2010). On the functional significance of Novelty-P3: Facilitation by unexpected novel sounds. Biological Psychology, 83(2), 143-152. doi:10.1016/j.biopsycho.2009.11.012

Schröger, E. (1996). A neural mechanism for involuntary attention shifts to changes in auditory stimulation. Journal of Cognitive Neuroscience, 8(6), 527-539.

Schröger, E., \& Wolff, C. (1998a). Attentional orienting and reorienting is indicated by human event-related brain potentials. Neuroreport, 9(15), 3355-3358.

Schröger, E., \& Wolff, C. (1998b). Behavioral and electrophysiological effects of taskirrelevant sound change: A new distraction paradigm. Cognitive Brain Research, 7(1), $71-87$.

Wetzel, N., Schröger, E., \& Widmann, A. (in press). The dissociation between the P3a eventrelated potential and behavioral distraction. Psychophysiology, doi:10.1111/psyp.12072 
Wetzel, N., Widmann, A., \& Schröger, E. (2012). Distraction and facilitation-two faces of the same coin? Journal of Experimental Psychology: Human Perception and Performance, 38(3), 664-674. doi:10.1037/a0025856

Winkler, I. (2007). Interpreting the Mismatch Negativity. Journal of Psychophysiology, 21(3), 147-163. doi:10.1027/0269-8803.21.34.147 


\section{FIGURE CAPTIONS}

Fig. 1 Group-mean d' sensitivity scores (left) and reaction times (right) for deviant and standard tones in the two conditions.

Fig. 2 Group-mean ERPs at $\mathrm{Fz}, \mathrm{Cz}, \mathrm{Pz}, \mathrm{Oz}$, and the average of the mastoid signals (CM) elicited by short (left column) and long (middle column) standard, deviant, Go and NoGo tones and the corresponding deviant-minus-standard difference waveforms (right column). The timing of the tones are represented in the form of black bars over the rulers. A vertical dashed line marks the timepoint at which the offset of the short tone could occur.

Fig. 3 Group-mean ERPs at Fz, Cz, Pz, Oz, and the average of the mastoid signals (CM) elicited by short and long standard and deviant stimuli (left column); the corresponding deviant-minus-standard difference waveforms (middle column), and the corresponding shortminus-long difference waveforms (right column). Arrows indicate the ERP components observable in the difference waveforms at $\mathrm{Cz}$ and $\mathrm{Fz}$. The timing of the tones are represented in the form of black bars over the rulers. A vertical dashed line marks the timepoint at which the offset of the short tone could occur.

Fig. 4 Group-mean ERP amplitudes measured in the P3a (left) and RON (right) time intervals for short, long, deviant and standard tones. The direction of the amplitude axes matches that of the ERP figures, that is, negative is upwards. 

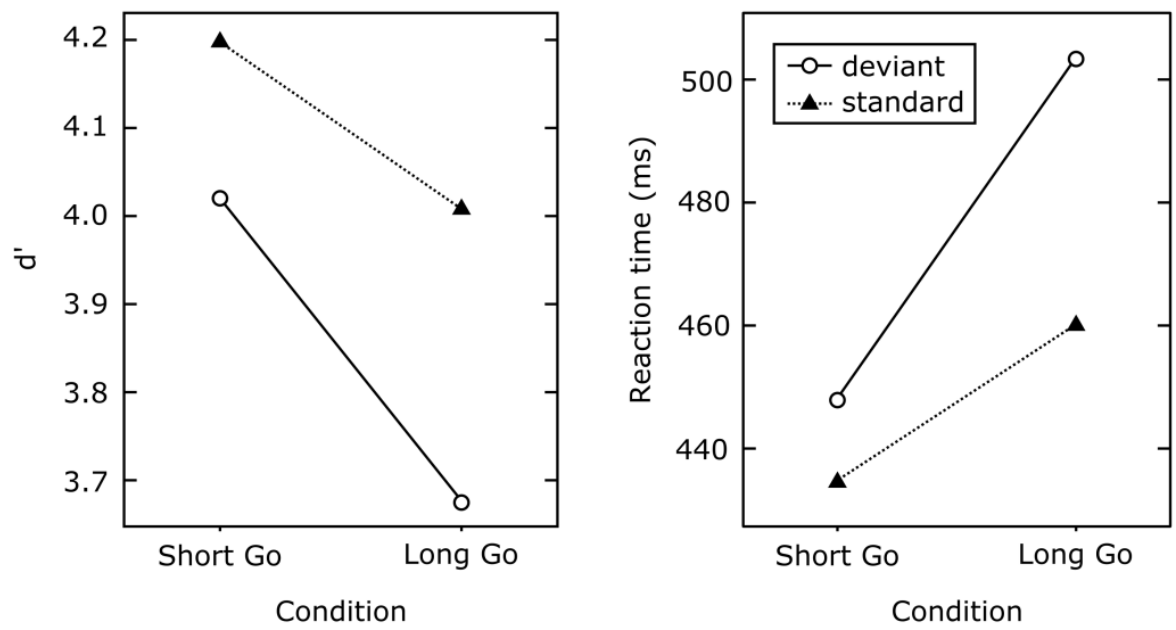

Fig. 1 Group-mean d' sensitivity scores (left) and reaction times (right) for deviant and standard tones in the two conditions. 

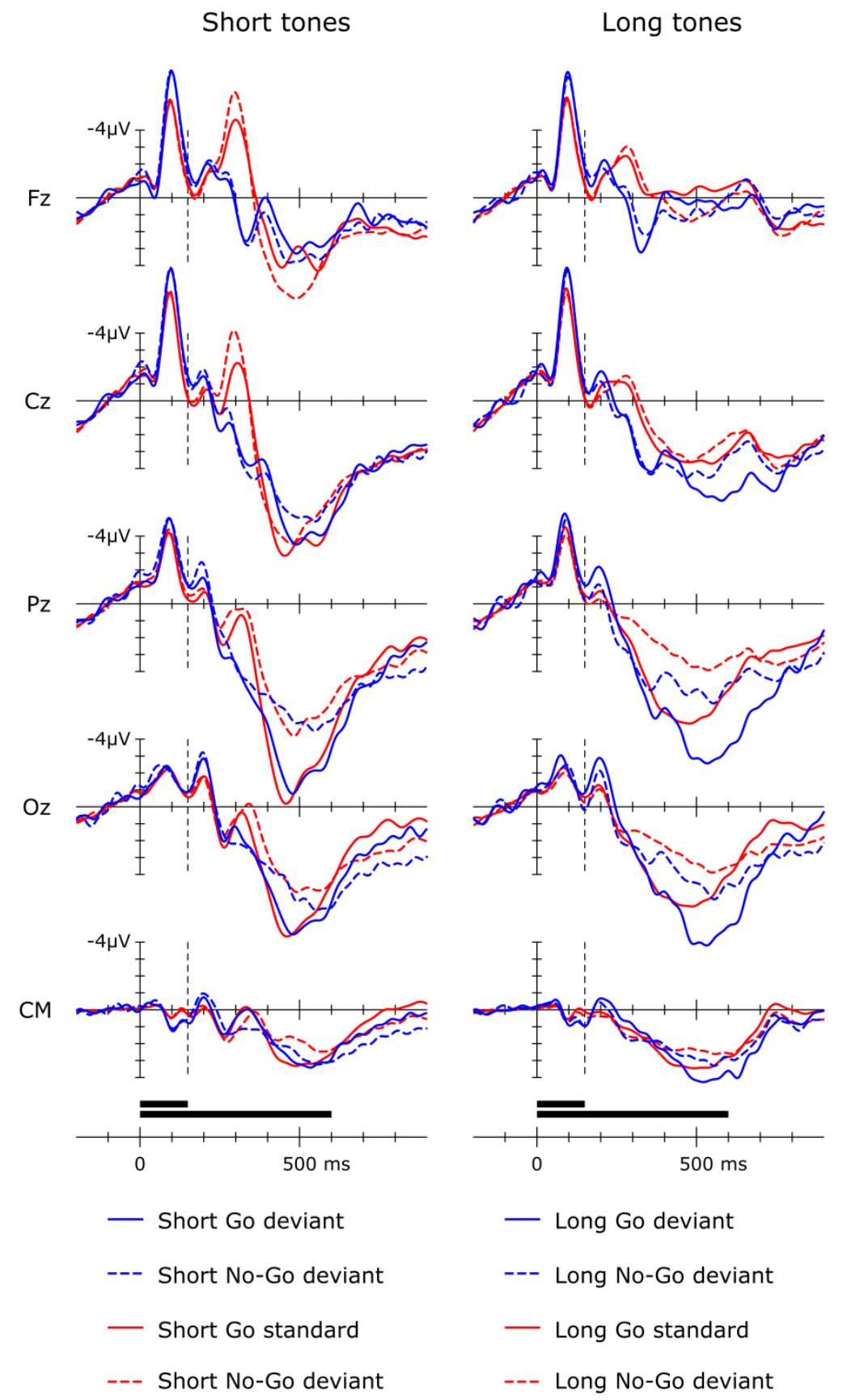
Deviant-minus-standard difference
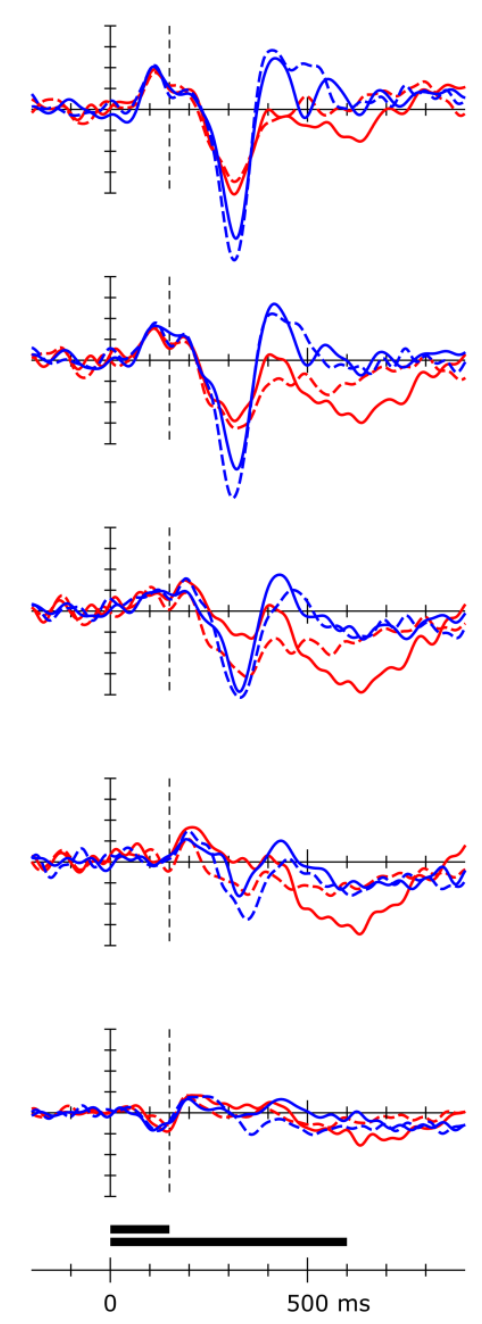

- Short Go difference

--- Short No-Go difference

- Long Go difference

--- Long No-Go difference

Fig. 2 Group-mean ERPs at $\mathrm{Fz}, \mathrm{Cz}, \mathrm{Pz}, \mathrm{Oz}$, and the average of the mastoid signals (CM) elicited by short (left column) and long (middle column) standard, deviant, Go and NoGo tones and the corresponding deviant-minus-standard difference waveforms (right column). The timing of the tones are represented in the form of black bars over the rulers. A vertical dashed line marks the timepoint at which the offset of the short tone could occur. 

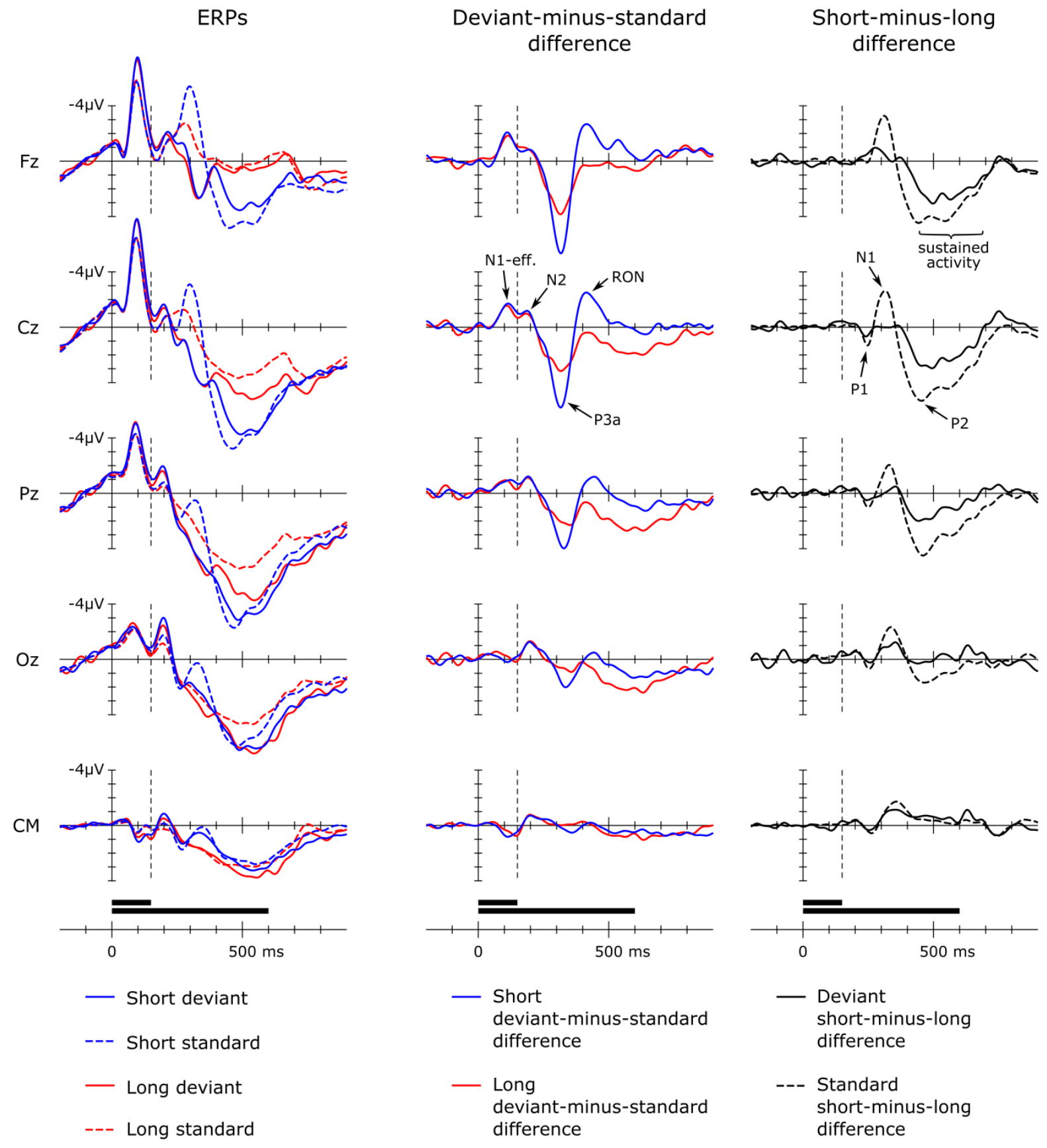
- Short deviant-minus-standard difference
— Long deviant-minus-standard

- Deviant short-minus-long difference difference

--- Standard short-minus-long difference

Fig. 3 Group-mean ERPs at $\mathrm{Fz}, \mathrm{Cz}, \mathrm{Pz}, \mathrm{Oz}$, and the average of the mastoid signals (CM) elicited by short and long standard and deviant stimuli (left column); the corresponding deviant-minus-standard difference waveforms (middle column), and the corresponding shortminus-long difference waveforms (right column). Arrows indicate the ERP components observable in the difference waveforms at $\mathrm{Cz}$ and $\mathrm{Fz}$. The timing of the tones are represented in the form of black bars over the rulers. A vertical dashed line marks the timepoint at which the offset of the short tone could occur. 

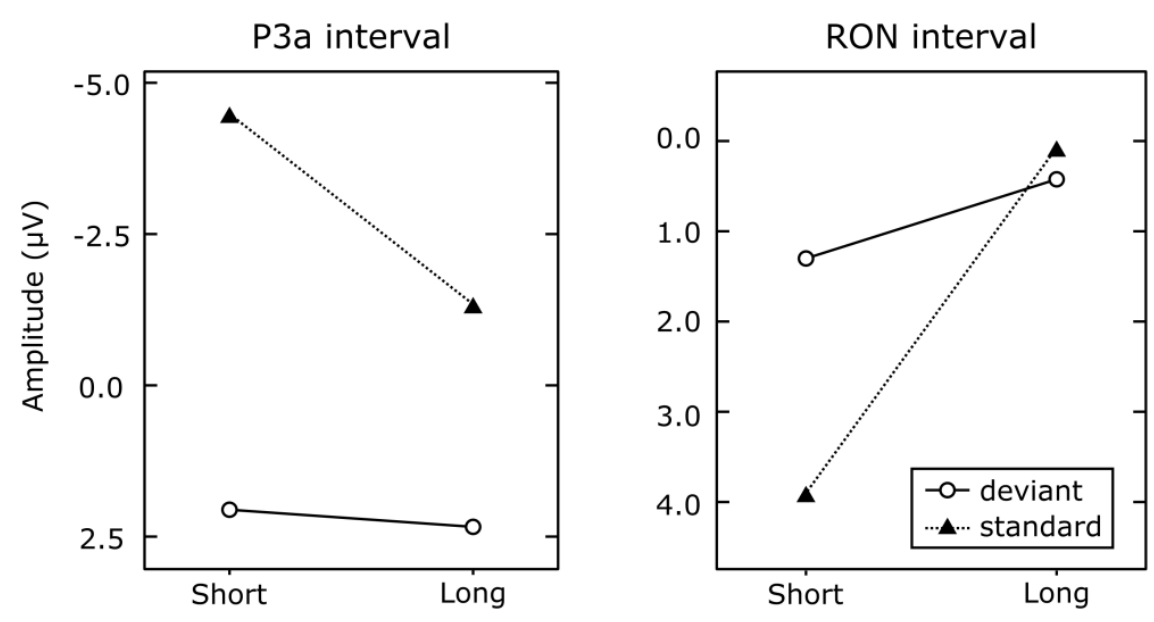

Fig. 4 Group-mean ERP amplitudes measured in the P3a (left) and RON (right) time intervals for short, long, deviant and standard tones. The direction of the amplitude axes matches that of the ERP figures, that is, negative is upwards. 